\title{
Hydrated hydroxide complex dominates the AIE property of nonconjugated polymeric luminophores
}

\author{
Taiqun Yang, a,b Jiafeng Zhou, ${ }^{\mathrm{b}}$ Bingqian Shan, ${ }^{\mathrm{b}}$ Lei Li, ${ }^{\mathrm{a}}$ Chun Zhu, ${ }^{\mathrm{a}}$ Chaoqun Ma, ${ }^{a}$ Guoqing Chen, ${ }^{\mathrm{a}}$ Kun \\ Zhang*b and Peng $\mathrm{Wu}^{\mathrm{b}}$
}

\begin{abstract}
Nontraditional intrinsic luminescence (NTIL) which always accompanied with aggregation-induced emission (AIE) features has received considerable attention due to their importance in the understanding of basic luminescence principle and potential practical applications. However, the rational modulation of the NTIL of nonconventional luminophores remains difficult, on account of the limited understanding of emission mechanisms. Herein, the emission colour of nonconjugated poly(methyl vinyl ether-alt-maleic anhydride) (PMVEMA) could be readily regulated from blue to red by controlling the alkalinity during the hydrolysis process. The nontraditional photoluminescence with AIE property was from the new formed $\mathrm{p}$-band state, resulting from the strong overlapping of $\mathrm{p}$ orbitals of the clustered $\mathrm{O}$ atoms though space interactions. Hydrated hydroxide complexes embedded in the entangled polymer chain make big difference on the clustering of $O$ atoms which dominates the AIE property of nonconjugated PMVEMA. These new insights into the photoemission mechanism of NTIL should stimulate additional experimental and theoretical studies and could benefit the molecular-level design of nontraditional chromophores for optoelectronics and other applications.
\end{abstract}

\section{Introduction}

Aggregation-induced emission (AIE) was a useful strategy and has been extensively investigated since the first observation in organic chromophores by Tang's group in 2001. ${ }^{1}$ Traditional AIE luminogens (T-AlEgens) are generally constructed with aromatic groups and conjugated subunits, which function as chromophore centers. ${ }^{2-4}$ However, the intricate synthesis process and toxic precursors limit their further application. Nontraditional AIE luminogens (NT-AIEgens) free of aromatic conjugate structure in their molecular structures and generally bearing electron rich moieties such as hydroxyl, amino, and carboxyl, etc. have received considerable attention due to their fundamental importance, promising applications. ${ }^{5,6}$ NT-AIEgens with typical structural features of no traditional chromophores gradually developed into a new type of AIE luminophores, including nature and synthetic small molecules, ${ }^{7-10}$ polymers ${ }^{11-16}$ and metal clusters. ${ }^{17-21}$ Among them, nonconjugated polymers, which are free of aromatic building blocks, have been intensively reported due to their unique clustering-triggered emission (CTE).

Although many studies have reported the nontraditional intrinsic luminescence (NTIL) in nonconjugated polymers, the rational modulation of the NTIL over the whole visible light range remains difficult. Most of the reported NTIL mainly focuses on the blue region and always with many defects including relatively low quantum yield (QY), limited production, etc. which further limited their practical application.22, 23 Poly(maleic anhydride-alt-vinyl acetate) (PMV) derivatives, a eco-friendly and low cost copolymer, were intensively studied for their unique NTIL properties. Tang et al. reported that PMV is strongly photoluminescent with the QY of $20 \%$ in organic solvents and the emission was attributed to anhydride cluster interacting in a through-space manner, while ring-opening reaction induced by water would result in remarkable decrease of photoluminescence (PL) which is detrimental for practical applications. ${ }^{24}$ Interestingly, Qiao et al. found that ring-opened PMV could also generate strong blue and red dual photoemission after interacting with acetone and metal hydroxide. ${ }^{25}$ They confirmed that the Na carboxylate group was the blue luminescence group with the excitation-dependent property, and the bulky carboxylic ester group was the red luminescence group with the excitation-independent property. Very recently, they realized the precisely regulation of the emission wavelength of hydrolyzed PMV from cyan to red by controlling the $\mathrm{pH}$ value. ${ }^{26,27}$

Despite great efforts have made to modulate the emission performance of nonconventional polymeric luminogens, their emission mechanism, especially for their emission center, is still controversial. Various assumptions have been suggested, including the oxidation of terminal group (unsaturated hydroxylamine center), ${ }^{28,}{ }^{29}$ crosslinking of the polymer chain (linear structure vs. hyperbranched structure), ${ }^{30}, 31$ hydrogen bond formation ${ }^{32}$ and electron delocalization. ${ }^{11,} 33$ Each view has its limitations which makes it difficult to reach a consensus. A recapitulative clusteringtriggered emission (CTE) mechanism was proposed by Yuan and Zhang and gradually accepted to elucidate the photoemission of nonconventional polymeric luminogens. ${ }^{34-36}$ The main principle of CTE was the close contact of the nonconventional chromophores leads to the overlapping of intra- and/or intermolecular electron clouds, forming an extended conjugation that generate fluorescence emissions. However, the fundamental principle of physical chemistry hidden behind the abnormal emission phenomena remains highly elusive, especially for the confirmation of the real emission center and the principle of through-space electron interactions. ${ }^{37}$ Herein, a new kind of PMV derivatives (PMVEMA) with NTIL properties was reported. Hydrated hydroxide complexes embedded in the entangled polymer chain make big difference on the AIE property of nonconjugated PMVEMA. By manipulating the delicate interactions between carbonyl groups and hydrated hydroxide complexes, we successfully achieve precise control of emission colours from blue to red. Combined with characterizations of steady-state and timeresolved spectroscopy, we identify the emission center of nonconventional polymeric luminogens, that is, the hydrated hydroxide complex assisted $p$-band intermediate state $\left(\mathrm{H}_{2} \mathrm{O} \bullet \mathrm{OH}^{-}-\right.$ PBIS) resulting from the strong overlapping of $p$ orbitals of the $O$ atoms of carbonyl and hydrated hydroxide though-space electron interactions. Different with the widely accepted CTE mechanism, we emphasize the predominant role of hydrated hydroxide. These new insights into the photoemission mechanisms of NTIL should stimulate additional experimental and theoretical research of nontraditional chromophores for optoelectronics and other applications. 


\section{Results and discussion}

These new kind of PMV derivatives with AIE properties were prepared by alcoholysis with PMVEMA under appropriate alkaline conditions (Scheme 1). The PMVEMA powder was pre-dispersed in anhydrous ethanol to form milky suspension with ultrasonic treatment. The formed PMVEMA/alcohol suspension disperse fast when dropped into $\mathrm{KOH}$ aqueous solution. As dropping is finished, yellow emulsion was obtained. After removing excess alcohol, the mixed emulsion becomes semi-transparent yellow solution in the daylight and the colour darkened with the increasing concentration of $\mathrm{KOH}$ (Fig. S1f, inserted left). The formed aqueous PMVEMA-K solution exhibits strong blue emission under UV irradiation (Fig. S1f, inserted right). The emission wavelength gradually red shifted from $\sim 400 \mathrm{~nm}$ to $\sim 535 \mathrm{~nm}$ with the dosage of $\mathrm{KOH}$ increased from $0 \mathrm{~g}$ to 2 g (Fig. S1, a-e). After drying, the polymer films showed tunable photoluminescent emission from blue to red under UV light (Fig. 1, a-f, inserted pictures). The 3D emission spectra of the photoluminescent polymer films were presented in Fig. 1a-f. It indicated that the emission wavelength gradually shifted to longer wavelength with the increasing dosage of $\mathrm{KOH}$. Noting that all the emission wavelengths are red shifted when dried from solution to film which implies the formation of a more compact structure in film state. The solidified film undergoes a dissolve-dry-dissolve process to evaluate the stability of the emission center. The original film and dissolved solution were named as the $1^{\text {st }}$-film and $1^{\text {st }}$-solution. The re-dried and re-dissolved samples were termed the $2^{\text {nd }}$-film and $2^{\text {nd }}$ solution. Different with the reported PMV derivatives, ${ }^{26}$ the PMVEMA-K film with intense red emission turn to yellow green when dissolved in water, as shown in Fig. S2. This was probably cause by the good water solubility of PMVEMA-K. When dissolved in water, the preformed emission structure was broken and a looser emission center formed due to the solvation effect. Noting that the red emission could recovered by re-drying. The emission wavelengths of $2^{\text {nd }}$-film and $2^{\text {nd }}$-solution shifted to long wavelength compared with $1^{\text {nd }}$-film and $1^{\text {nd }}$-solution, and the emission was intensified which implies an environment and assembly structure sensitive emission center.

The emission mechanism of this nonconventional polymeric luminogens with tenable emission wavelength was still elusive, especially for their real emission center. Qiao et. al. ascribed the metal carboxylate group to the blue emission center with the excitation-dependent property, and the bulk carboxylic ester group as the red emission center with the excitation-independent property. ${ }^{25}$ In addition, they found that the PMV solids could be transformed into red-emission reagents by simple heat-treatment and the red photoluminescence was attribute to the formation of isolated $\mathrm{C}=\mathrm{C}$ bonds. ${ }^{27}$ However, we find that the hydrolyzed PMVEMA-K without carboxylic ester group exhibit the same emission
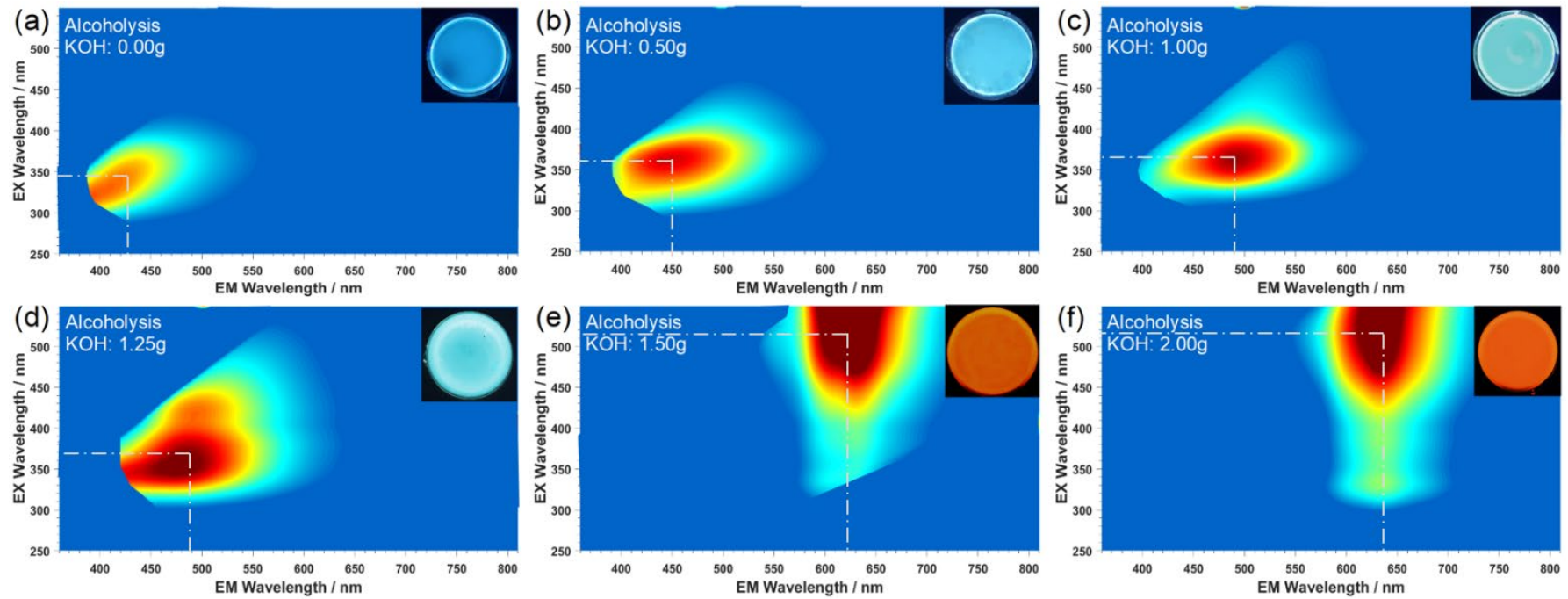

Figure 1. The 3D photoluminescence spectra of PMVEMA-K films prepared with different dosage of $\mathrm{KOH}$ (alcoholysis). Insets show corresponding photographs under UV light. Noting that the background scattering has been subtracted in the 3D photoluminescence spectra. 


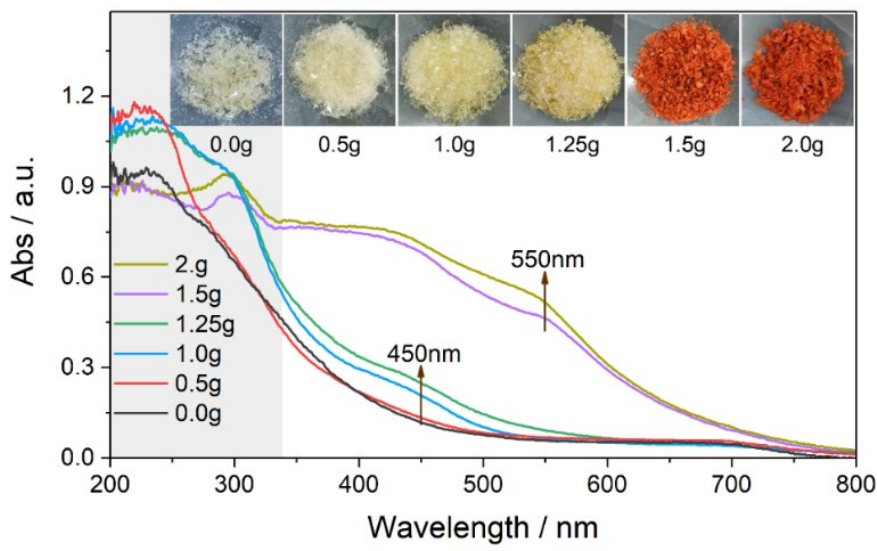

Figure 2. UV-Vis diffuse reflection spectra of as-prepared PMVEMA$\mathrm{K}$ powders with different dosage of $\mathrm{KOH}$ (alcoholysis).

properties as alcoholyzed PMVEMA-K. As shown in Fig. S3, these hydrolyzed PMVEMA-K films also showed tunable photoemission from blue to red with the increasing dosage of $\mathrm{KOH}$. So, what is the real emission center for this $\mathrm{pH}$ controlled nontraditional intrinsic luminescence of nonconventional polymeric luminogens? The emission spectra of alcoholyzed and hydrolyzed PMVEMA-K films indicated that the emission is mainly consisted of two typical emission bands (blue: $\sim 420, \sim 450, \sim 465, \sim 485 \mathrm{~nm}$, red: $\sim 580, \sim 605$, $\sim 625, \sim 650 \mathrm{~nm}$ ), as shown in Fig. S4. The relative intensity of each emission peak could be regulated by controlling the dosage of $\mathrm{KOH}$. The UV-Vis diffuse reflection spectra showed that these alcoholyzed and hydrolyzed PMVEMA-K powders had significant absorptions in UV and visible regions (Fig. 2 and Fig. S5). The broad absorption peaks below $350 \mathrm{~nm}$ were ascribed to $\pi-\pi^{*}$ and $n-\pi^{*}$ transitions of $C=0$ bonds in aggregated state due to the conjugation or delocalization of electrons between neighbouring carbonyl groups in the polymer chain. ${ }^{17,20}$ With the increase of $\mathrm{KOH}$ usage, two broad shoulder peaks at $\sim 450 \mathrm{~nm}$ and $550 \mathrm{~nm}$ appeared and intensified, which corresponds to their 3D emission spectra and implies the formation of new emission center caused by aggregation. Noting that even for the excited state transition of clustered carbonyl groups, the absorption at more than $400 \mathrm{~nm}$ usually cannot be distinguished. ${ }^{17,20}$ In addition, we find that the water content of PMVEMA-K powders gradually increased with the increasing dosage of $\mathrm{KOH}$. Noting that two kinds of water molecules (the physical and chemical adsorbed water) are presented in the aggregation structure and the physical adsorbed water molecules were removed by vacuum drying before doing thermogravimetric measurements. As indicated in Fig. 3, all these asprepared PMVEMA-K powders contain a certain amount of bound water. The decomposition temperature of bound water gradually increased with the increasing dosage of $\mathrm{KOH}$ from $0 \mathrm{~g}$ to $2 \mathrm{~g}$. Simultaneously, the bound water content was dramatically increased from $2.5 \%$ to $14.6 \%$. Combined with further conducted spectroscopic analysis, we can conclude that the hydrated hydroxide complex dominates the AIE property of nonconjugated poly(methyl vinyl ether-alt-maleic anhydride). It is reasonable that, in the confined nanospace, the clustering of $\mathrm{O}$ atoms from carbonyl and hydrated hydroxide through-space electron interactions, namely, overlapping of these lone pair ( $n$ ) electrons of $p$ orbitals, extends the conjugation. Thus, the corresponding intense absorption bands at more than 400 $\mathrm{nm}$ are observed with subsequent intense photoemission (Fig. 2).

A common feature for these nonconjugated polymers with AIE properties is that their polymer chains always contain electron-rich heteroatoms, including nitrogen $(\mathrm{N})$, oxygen $(\mathrm{O})$, sulfur $(\mathrm{S})$ and phosphorous $(\mathrm{P})$, and so forth, which could promote the electron communication through-space electron interactions. A descriptive hydrated hydroxide complex assisted $\mathrm{p}$-band intermediate state $\left(\mathrm{H}_{2} \mathrm{O} \cdot \mathrm{OH}^{-}-\mathrm{PBIS}\right)$ model was proposed to explain the origin of the
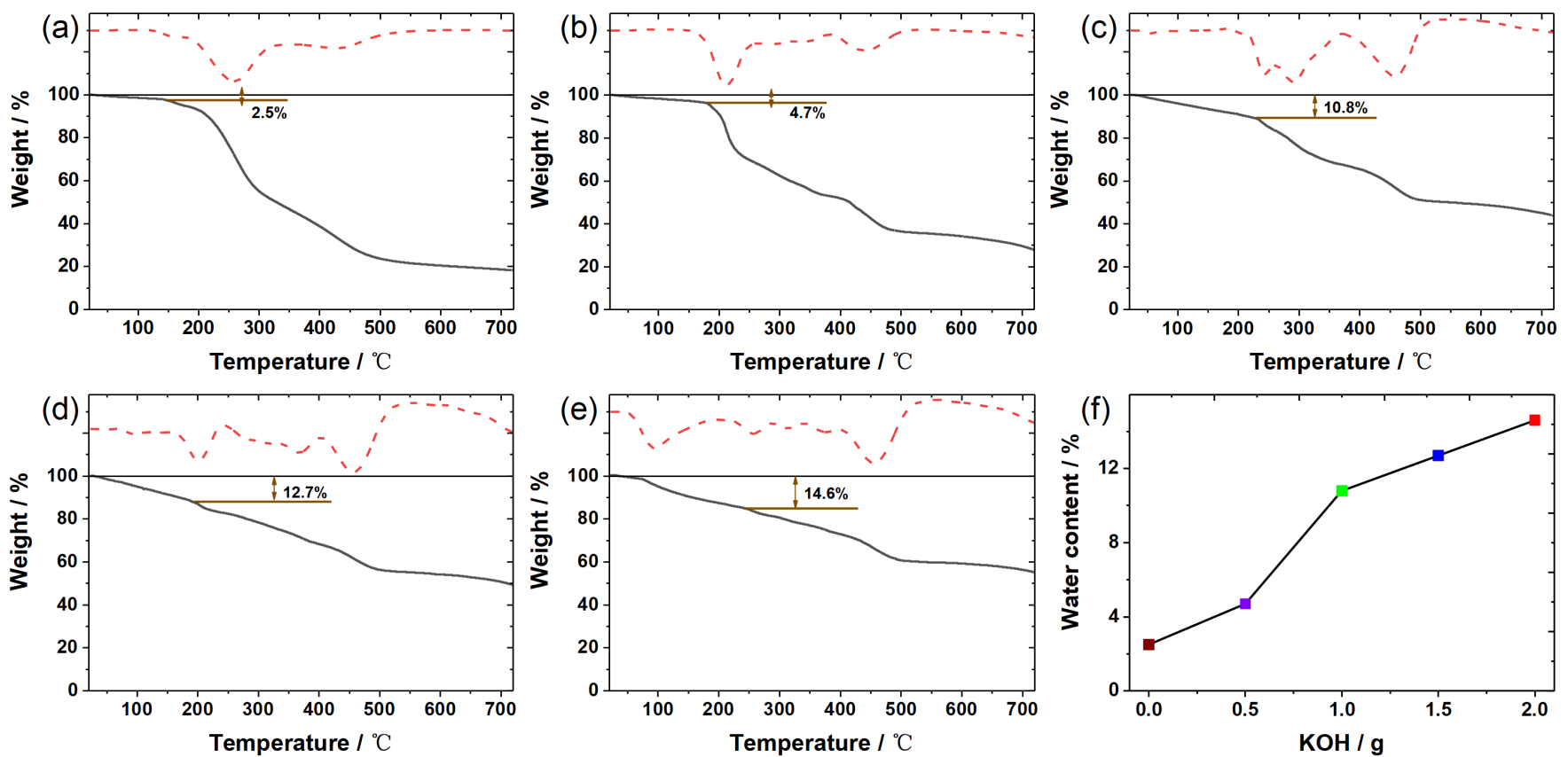

Figure 3. (a-e) Thermogravimetric (TG) and differential thermogravimetric (DTG) curves of as-prepared PMVEMA-K powders with different usage of $\mathrm{KOH}$. Noting that these samples were processed at $80{ }^{\circ} \mathrm{C}$ in an oven to remove the physical adsorbed water before doing thermogravimetric measurements. (f) Correlations of the water content of PMVEMA-K powder versus dosage of $\mathrm{KOH}$. 


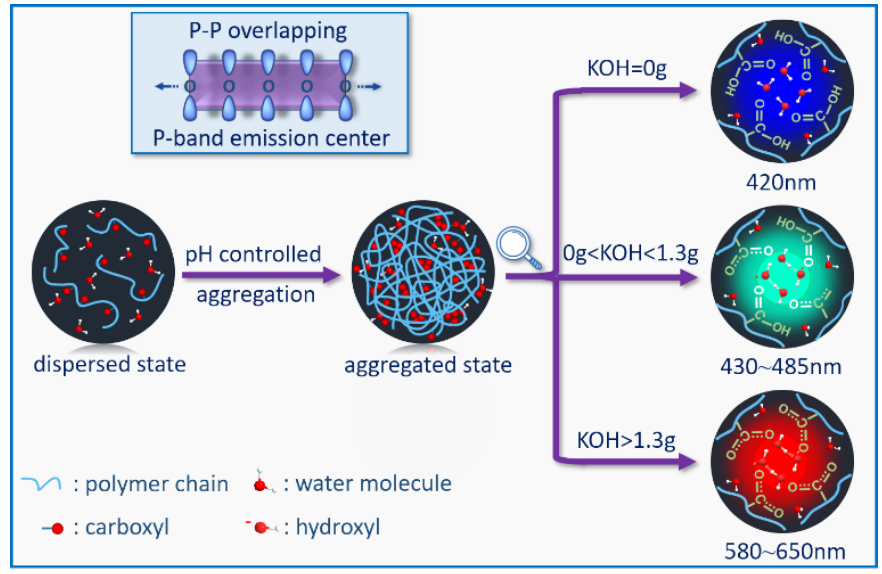

Figure 4. Hydrated hydroxide complex assisted $p$-band intermediate state $\left(\mathrm{H}_{2} \mathrm{O} \bullet \mathrm{OH}^{-}-\mathrm{PBIS}\right)$ dominates photoluminescence of polymeric luminogens. Schematic illustration of the alkalinity controlled AIE properties of PMVEMA-K samples. The $p$-band emission center is formed by the overlapping of $p$-orbitals of oxygen atoms in the confined nanospace.

$\mathrm{pH}$-controlled photoemission of nonconventional polymeric luminogens. As shown in Fig. 4, these water-soluble polymer chains gradually contracted during the dehydration process under certain alkalinity condition. Finally, a new dense aggregation structure formed and the hydrated hydroxide complexes were locked in the local confined nanospace. At the nanoconfined space, the adjacent $O$ atoms locally interact with each other to form Rydberg matter-like clusters by the overlapping of p-orbitals. ${ }^{38,39}$ The delocalization of the high-energy electrons produces a new overall lower energy state, the so-called $\mathrm{p}$-band intermediate state $(\mathrm{PBIS}),{ }^{20}$ which acts as the real emission center of nonconventional polymeric luminogens and dominates their AIE properties. The hydrated hydroxide complexes make big difference on the formation of $\mathrm{p}$-band intermediate sate. Firstly, it governs the aggregation structure of the carbonyl clusters by hydrogen bond interaction. Secondly, these $\mathrm{O}$ atoms of hydrated hydroxide complexes also contribute to the $\mathrm{p}$-band emission center. So, the emission properties of PMVEMA-K could be adjusted by controlling the $\mathrm{pH}$ value. (1) $\mathrm{KOH}=0 \mathrm{~g}$. When no $\mathrm{KOH}$ was added during the hydrolysis process, most of these carboxyl groups are

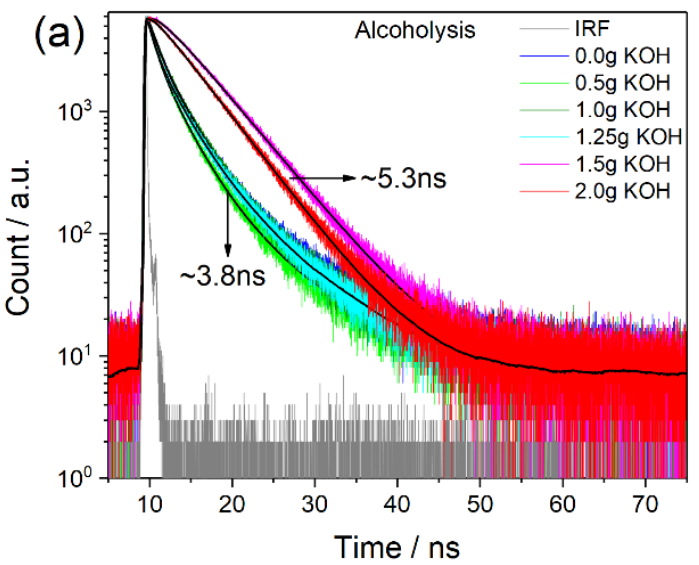

protonated (Fig. S6, red curve) and few hydroxyls are presented in the nanospace. The emission center was formed by the clustering of carboxyl groups and water molecules with blue photoluminescence which often observed in Natural and synthetic nontraditional luminogens. ${ }^{5}, 33,36,40,41$ (2) $0 \mathrm{~g}<\mathrm{KOH}<1.3$ g. When $\mathrm{KOH}$ was introduced into the system, the carboxyl groups start to deprotonate and water molecules are presented as hydrated hydroxide complexes. The emission center becomes the clustering of partially deprotonated carboxyl groups and hydrated hydroxide complexes with tunable emission wavelength from 430 to $485 \mathrm{~nm}$. (3) $\mathrm{KOH}>1.3$ g. When the dosage of $\mathrm{OH}$ exceed $1.3 \mathrm{~g}$, the carboxyl groups are totally deprotonated (Fig. S6, blue curve). The emission center turns into the clustering of deprotonated carboxyl groups and hydrated hydroxide complexes with tunable emission wavelength from 580 to $650 \mathrm{~nm}$. Based on our PBIS model, the p-band state was mainly correlated with the distance between adjacent oxygen atoms. ${ }^{20}$ So by controlling the alkalinity during the hydrolysis process of PMVEMA, we successfully achieved the precise regulation of the distance between these two adjacent oxygen atoms at the molecule level.

Combined with these results, it gets clear that two kinds of emission center with blue and red emission region were constructed with the assistant of hydrated hydroxide complexes. This consideration is supported by studying the luminescence lifetime of as-prepared PMVEMA-K films with different dosage of $\mathrm{KOH}$. As demonstrated in Fig. 5, the time-resolved fluorescence measurements indicate that the average lifetime of these asfabricated prepared PMVEMA-K films with dosage of $\mathrm{KOH}$ less than $1.3 \mathrm{~g}$ is $\sim 3.8 \mathrm{~ns}$ (fitted by three exponents) and the predominant lifetime is in 1 3 ns range (Table s1). When the dosage of $\mathrm{KOH}$ exceeds $1.3 \mathrm{~g}$, the average lifetime of these as-fabricated prepared PMVEMA-K films increases to $\sim 5.3 \mathrm{~ns}$ (fitted by single exponent). The simplification of emission lifetime components implies that a compact and uniform emission center was formed under high alkaline conditions. According to the $\mathrm{H}_{2} \mathrm{O}^{\circ} \mathrm{OH}^{-}-\mathrm{PBIS}$ model and considering the orientation of $\mathrm{p}$ orbitals in the space, it could generate multiple $p$-band state in the same clustering assembly structure with identical O-O distance, which was further verified by the photoluminescence spectra. As shown in Fig. S4, a and c, the emission spectra of PMVEMA-K films exhibit multiple emission peak

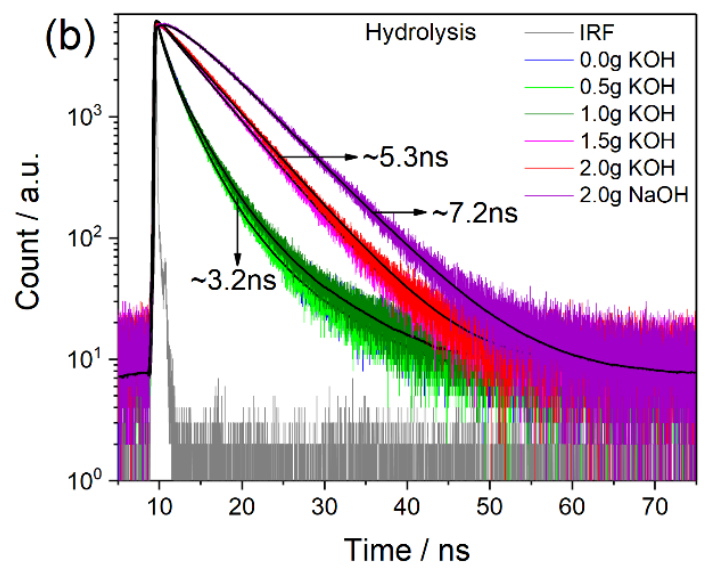

Figure 5. Time-resolved luminescence decay profiles of as-prepared PMVEMA-K films with different dosage of KOH. (left: alcoholysis, right: hydrolysis) 

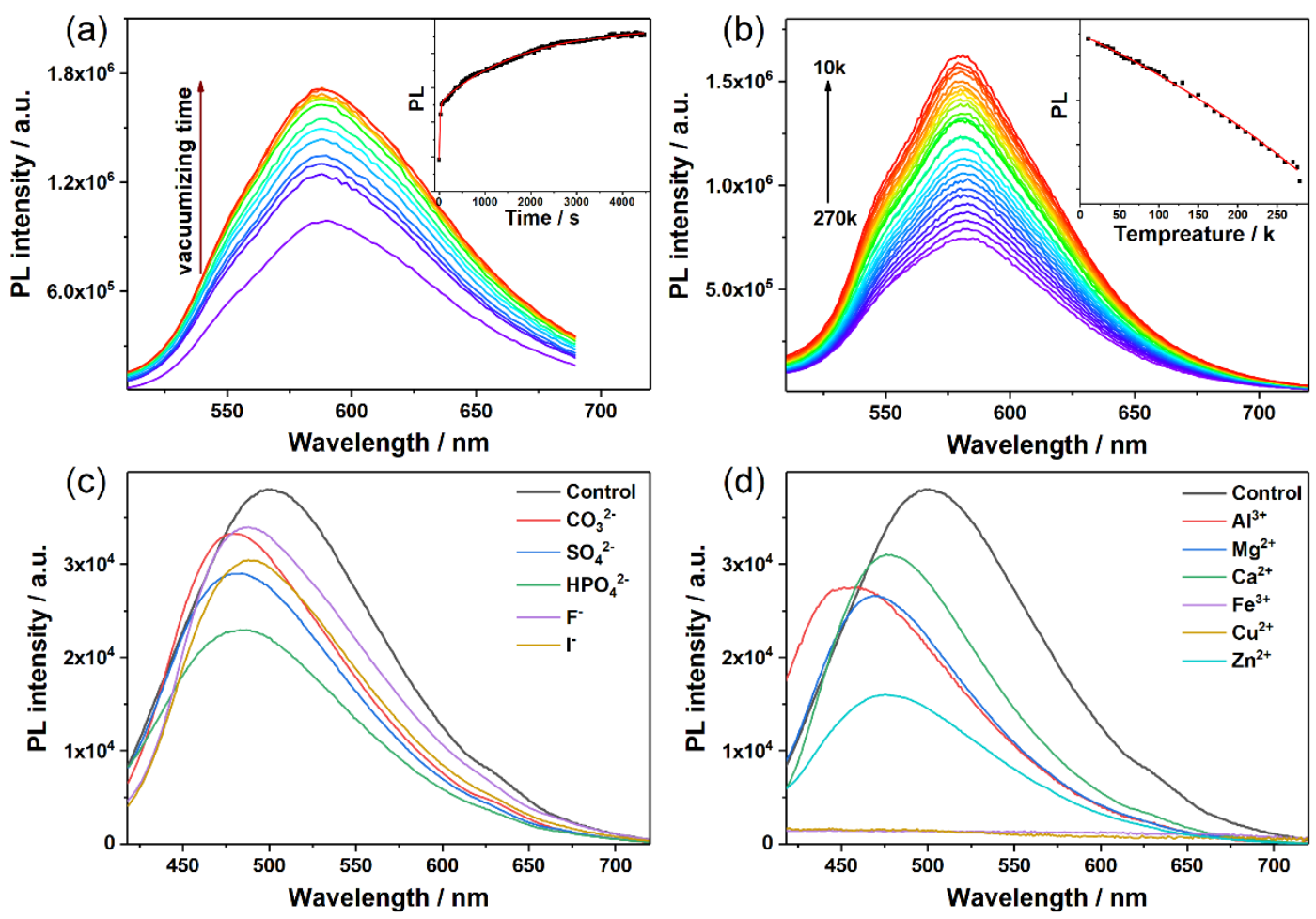

Figure 6. $(a, b)$ Emission spectra of as-fabricated PMVEMA-K films with different anions and cations. (c) PL increment of PMVEMA-K films with increasing time of vacuumizing. Inset shows the corresponding PL intensity augment during the dehydration process. (d) Temperaturedependent PL enhancement of PMVEMA-K. Inset shows the PL intensity is exponentially increased with decreasing temperature from $270 \mathrm{k}$ to $10 \mathrm{k}$. (sample a and b: alcoholysis, $\mathrm{KOH}=1 \mathrm{~g}$; sample $\mathrm{c}$ and $\mathrm{d}$ : hydrolysis, $\mathrm{KOH}=1.5 \mathrm{~g}$ )

under a certain excitation wavelength. Differing from the localized excitation of conventional chromophore, the emission of nontraditional polymeric luminogens exhibit topological excitation properties which induced by the dynamic combinations of $p$ orbitals of two adjacent oxygen atoms in different directions though-space interactions.

Based on our $\mathrm{H}_{2} \mathrm{O} \cdot \mathrm{OH}^{-}-$PBIS model, the photoluminescence of PMVEMA-K should be sensitive to the adsorbed water molecules including physical and chemical adsorbed water. To investigate the influence of physical adsorbed water on the photoemission properties of polymeric luminogens, we supervise the emission spectra of PMVEMA-K powders during the dehydration process by vacuumizing. In Fig. 6a, with increasing time of vacuumizing, the photoluminescence intensity exponentially increased. The PL intensity sharply increased $\sim 30 \%$ in the first minute and then slowly increased $\sim 30 \%$ in the next hour and then sustained which implies a dehydration process of unstable physical absorbed water. Interestingly, the increased PL of PMVEMA-K would be recovered by water absorption and the PL exhibits reversible evolution upon dehydration/hydration process in vacuum/atmosphere conditions (Fig. S7). This is consistent with experimental observations that the PL of PMVEMA-K gradually weakened when exposed to atmospheric conditions with high humidity. Too much physical adsorbed water will destroy the structure of emission center by hydrogen bond interaction which lead to the decrease of PL intensity. For the chemical adsorbed water, it involves in the emission center and is difficult to remove, as evidenced by thermogravimetric analysis (Fig. 3). To further verify the validity of $\mathrm{H}_{2} \mathrm{O} \cdot \mathrm{OH}^{-}-\mathrm{PBIS}$ model, we modified the photoluminescence signal of PMVEMA-K by regulating the hydrogen bond. As shown in Fig. 6, c and d, Fig. S8 and Fig. S9, the $\mathrm{PL}$ intensity was decreased when introducing other ions to destroy the hydrogen bond. Owing to the salt-out effect, the dosing of Hofmeister series ions perturbs the interaction between hydrated hydroxyl complexes and surrounding carbonyl groups, leading to the damage of preformed emission center. Consequently, PL emission of PMVEMA-K was decreased. The PL of PMVEMA-K was temperaturedependent due to their susceptible emission center. As shown in Fig. $6 \mathrm{~d}$, the PL intensity of PMVEMA-K powders significantly increased with temperature dropping which implies the restriction of rotations and vibrations of the hydrated hydroxide complexes assisted emission center. From these results, we can conclude that hydrated water complexes are crucial for stabilize the emission center of nonconventional polymeric luminogens and these results provide an effective method for controlling these ubiquitous weak interactions. The previously reported abnormal emission phenomena for nontraditional AIE luminogens now can be readily understood due to the formation of diversity of PBIS with the assistance of hydrated hydroxide complexes at the confined nanospace, including $\mathrm{pH}$ and thermo sensitive photoemission, ${ }^{26,} 27,42$ solvatochromic effect, ${ }^{24,} 25$ oxidation-controlled photoemission ${ }^{28,43}$ and molecular weightdependent fluorescence. ${ }^{16}$ 


\section{Conclusions}

In summary, the nontraditional photoluminescence of PMVEMA was readily modulated from blue to red using a $\mathrm{pH}$-controlled hydrolysis strategy. Based on the solid experimental evidences, we first demonstrated that hydrated hydroxide complexes conducted the construction of the emission center which dominates the AIE property of nonconjugated polymeric luminogens. A hydrated hydroxide complex assisted $p$-band intermediate state $\left(\mathrm{H}_{2} \mathrm{O} \cdot \mathrm{OH}^{-}-\right.$ $\mathrm{PBIS}$ ) model was proposed to explain the origin of the $\mathrm{pH}$-controlled photoemission. The PL of nonconventional polymeric luminogens was from the intermediate $p$-band state due to the overlapping of $p$ orbitals with high-energy lone-pair electrons through-space electron interactions. The hydrated hydroxide complexes involve in the construction of the $p$-band emission center, as well as regulate the microstructure of the luminescent center at molecular level by hydrogen bond interaction. The dynamic property of $\mathrm{H}_{2} \mathrm{O} \bullet \mathrm{OH}^{-}-\mathrm{PBIS}$ makes it susceptible to the microenvironment, especially for the $\mathrm{pH}$ condition which provides an avenue for modulating the photoluminescence performance of nontraditional polymeric luminogens. This proposed $\mathrm{H}_{2} \mathrm{O} \bullet \mathrm{OH}^{-}-$PBIS model is not only capable for explaining the peculiar optoelectronic properties of nontraditional AIE luminogens, $, 22,36,44$ but also provides completely new insights for the understanding of the photoemission process of proteins. $^{45-47}$

\section{Author Contributions}

K.Z. and T.Y. designed the experiments. T.Y. performed the main experiments. J.Z. and B.S. performed the thermogravimetric (TG) measurements. L.L. performed the TCSPC measurements. C.Z. and C.M. performed the vacuum fluorescence experiment. K.Z. and T.Y. modified the PBIS model and proposed the $\mathrm{H}_{2} \mathrm{O}^{\circ} \mathrm{OH}^{-}-\mathrm{PBIS}$ model which is not only capable for explaining the peculiar photoluminescence properties of nontraditional AIE luminogens, but also provides completely new insights for the understanding of the photoemission process of proteins. T.Y. designed the figures for the manuscript with the assistance of G.C. and P.W. T.Y., K.Z. and P.W. prepared the manuscript with contributions from all co-authors.

\section{Conflicts of interest}

There are no conflicts to declare.

\section{Acknowledgements}

The authors thank Prof. Jinliang Qiao and Dr. ChenXi Hu for their helpful suggestions on material synthesis. This work was supported by the NSFC (21872053, 2157307 and 461378037), the Science and Technology Commission of Shanghai Municipality (19520711400), the CAS key laboratory of LowCoal Conversion Science \& Engineering (KLLCCSE-201702), and the JORISS program, the sub-project of the National Key Research and Development Program of china (2018YFC1604204), the national first-class discipline program of Food Science and Technology (JUFSTR20180302), the Key
Research and Development Program of Jiangsu Province (BE2020756).

\section{Notes and references}

1 J. Luo, Z. Xie, J. W. Lam, L. Cheng, H. Chen, C. Qiu, H. S. Kwok, X. Zhan, Y. Liu, D. Zhu and B. Z. Tang, Chem. Commun., 2001, DOI: 10.1039/b105159h, 1740-1741.

2 J. Mei, N. L. C. Leung, R. T. K. Kwok, J. W. Y. Lam and B. Z. Tang, Chem. Rev., 2015, 115, 11718-11940.

3 Y. Chen, J. W. Y. Lam, R. T. K. Kwok, B. Liu and B. Z. Tang, Mater. Horiz., 2019, 6, 428-433.

4 R. Hu, A. Qin and B. Z. Tang, Prog. Polym. Sci., 2020, 100.

5 Y. Wang, Z. Zhao and W. Z. Yuan, Chempluschem, 2020, 85, 1065-1080.

6 H. Zhang, Z. Zhao, P. R. McGonigal, R. Ye, S. Liu, J. W. Y. Lam, R. T. K. Kwok, W. Z. Yuan, J. Xie, A. L. Rogach and B. Z. Tang, Mater. Today, 2020, 32, 275-292.

7 X. Chen, W. Luo, H. Ma, Q. Peng, W. Z. Yuan and Y. Zhang, Sci. China Chem., 2017, 61, 351-359.

8 S. Zheng, T. Zhu, Y. Wang, T. Yang and W. Z. Yuan, Angew. Chem. Int. Ed., 2020, 59, 1-6.

9 Q. Zhou, T. Yang, Z. Zhong, F. Kausar, Z. Wang, Y. Zhang and W. Z. Yuan, Chem. Sci., 2020, 11, 2926-2933.

10 X. Dou, Q. Zhou, X. Chen, Y. Tan, X. He, P. Lu, K. Sui, B. Z. Tang, Y. Zhang and W. Z. Yuan, Biomacromolecules, 2018, 19, 20142022.

11 Z. Dong, H. Cui, Y. Wang, C. Wang, Y. Li and C. Wang, Carbohyd. Polym., 2020, 227, 115338.

12 Q. Zhou, Z. Wang, X. Dou, Y. Wang, S. Liu, Y. Zhang and W. Z. Yuan, Mater. Chem. Front., 2019, 3, 257-264.

13 C. Shang, Y. Zhao, J. Long, Y. Ji and H. Wang, J. Mater. Chem. C, 2020, 8, 1017-1024.

14 B. Liu, B. Chu, Y. L. Wang, Z. Chen and X. H. Zhang, Adv. Optical Mater., 2020, DOI: 10.1002/adom.201902176.

15 Z. Dong, Y. Wang, C. Wang, H. Meng, Y. Li and C. Wang, Adv. Healthcare Mater., 2020, 9, e2000419.

16 C. Shang, N. Wei, H. Zhuo, Y. Shao, Q. Zhang, Z. Zhang and H. Wang, J. Mater. Chem. C, 2017, 5, 8082-8090.

17 T. Yang, S. Dai, S. Yang, L. Chen, P. Liu, K. Dong, J. Zhou, Y. Chen, H. Pan, S. Zhang, J. Chen, K. Zhang, P. Wu and J. Xu, J. Phys. Chem. Lett., 2017, 8, 3980-3985.

18 Z. Luo, X. Yuan, Y. Yu, Q. Zhang, D. T. Leong, J. Y. Lee and J. Xie, J. Am. Chem. Soc., 2012, 134, 16662-16670.

19 M. Sugiuchi, J. Maeba, N. Okubo, M. Iwamura, K. Nozaki and K. Konishi, J. Am. Chem. Soc., 2017, 139, 17731-17734.

20 T. Yang, B. Shan, F. Huang, S. Yang, B. Peng, E. Yuan, P. Wu and K. Zhang, Commun. Chem., 2019, 2, 132.

21 T. Q. Yang, B. Peng, B. Q. Shan, Y. X. Zong, J. G. Jiang, P. Wu and K. Zhang, Nanomaterials, 2020, 10, 261.

22 S. Tao, S. Zhu, T. Feng, C. Zheng and B. Yang, Angew. Chem. Int. Ed., 2020, 59, 9826-9840.

23 Q. Li, Y. Tang, W. Hu and Z. Li, Small, 2018, 14, e1801560.

24 E. Zhao, J. W. Y. Lam, L. Meng, Y. Hong, H. Deng, G. Bai, X. Huang, J. Hao and B. Z. Tang, Macromolecules, 2015, 48, 6471.

25 C. Hu, Z. Guo, Y. Ru, W. Song, Z. Liu, X. Zhang and J. Qiao, Macromol. Rapid Commun., 2018, 39, e1800035.

26 C. Hu, Y. Ru, Z. Guo, Z. Liu, J. Song, W. Song, X. Zhang and J. Qiao, J. Mater. Chem. C, 2019, 7, 387-393.

27 Z. Guo, Y. Ru, W. Song, Z. Liu, X. Zhang and J. Qiao, Macromol. Rapid Commun., 2017, 38, 1700099.

28 W. I. Lee, Y. Bae and A. J. Bard, J. Am. Chem. Soc., 2004, 126, 8358-8359.

29 S. Y. Lin, T. H. Wu, Y. C. Jao, C. P. Liu, H. Y. Lin, L. W. Lo and C. S. Yang, Chem. Eur. J., 2011, 17, 7158-7161. 
30 L. Fang, C. Huang, G. Shabir, J. Liang, Z. Liu and H. Zhang, ACS Macro Lett., 2019, 8, 1605-1610.

31 C. Shang, Y. Zhao, N. Wei, H. Zhuo, Y. Shao and H. Wang, Macromol. Chem. Phys., 2019, 220, 1900324.

32 D. Pinotsi, L. Grisanti, P. Mahou, R. Gebauer, C. F. Kaminski, A. Hassanali and G. S. Kaminski Schierle, J. Am. Chem. Soc., 2016, 138, 3046-3057.

33 R. Ye, Y. Liu, H. Zhang, H. Su, Y. Zhang, L. Xu, R. Hu, R. T. K. Kwok, K. S. Wong, J. W. Y. Lam, W. A. Goddard and B. Z. Tang, Polym. Chem., 2017, 8, 1722-1727.

34 Y. Gong, Y. Tan, J. Mei, Y. Zhang, W. Yuan, Y. Zhang, J. Sun and B. Z. Tang, Sci. China Chem., 2013, 56, 1178-1182.

35 Q. Zhou, B. Cao, C. Zhu, S. Xu, Y. Gong, W. Z. Yuan and Y. Zhang, Small, 2016, 12, 6586-6592.

36 W. Zhang Yuan and Y. Zhang, J. Polym. Sci. Polym. Chem., 2017, $55,560-574$

37 R. Hoffmann, Acc. Chem. Res., 1971, 4, 1-9.

38 E. K. Campbell, M. Holz, D. Gerlich and J. P. Maier, Nature, 2015, 523, 322-323.

39 L. Holmlid, Mon. Not. R. Astron. Soc., 2008, 384, 764-774.

40 L.-L. Du, B.-L. Jiang, X.-H. Chen, Y.-Z. Wang, L.-M. Zou, Y.-L. Liu, Y.-Y. Gong, C. Wei and W.-Z. Yuan, Chinese J. Polym. Sci., 2019, 37, 409-415.

41 Y. Wang, X. Bin, X. Chen, S. Zheng, Y. Zhang and W. Z. Yuan, Macromol. Rapid Commun., 2018, 39, e1800528.

42 Q. Jiang, Y. Du, Y. Zhang, L. Zhao, L. Jiang, W. Huang, H. Yang, $X$. Xue and B. Jiang, J. Polym. Sci., 2020, 58, 2718-2727.

43 Z. Zhao, X. Chen, Q. Wang, T. Yang, Y. Zhang and W. Z. Yuan, Polym. Chem., 2019, 10, 3639-3646.

44 X. Li, M. Li, M. Yang, H. Xiao, L. Wang, Z. Chen, S. Liu, J. Li, S. Li and T. D. James, Coordin. Chem. Rev., 2020, 418, 213358.

45 H. NIWA, S. INOUYE, T. HIRANO, T. MATSUNO, S. KOJIMA, M. KUBOTA, M. OHASHI and F. I. TSUJI, Proc. Natl. Acad. Sci. USA 1996, 93, 13617-13622.

46 R. Bizzarri, M. Serresi, S. Luin and F. Beltram, Anal. Bioanal. Chem., 2009, 393, 1107-1122.

47 T. D. Craggs, Chem. Soc. Rev., 2009, 38, 2865-2875. 